\title{
Teor e composição do óleo essencial de patchouli (Pogostemon cablin (Blanco) Benth.) após diferentes tempos de secagem em estufa e temperatura ambiente
}

STORCK, R.C. ${ }^{1 *}$; DESCHAMPS, C. ${ }^{1}$

'Universidade Federal do Paraná, Departamento de Fitotecnia e Fitossanitarismo. Rua dos Funcionários, 1540, Curitiba -PR, CEP 81531-990. *Autor para correspondência: rafaellencstorck@yahoo.com.br

RESUMO: Patchouli é uma espécie aromática pertencente à família Lamiaceae que possui óleo essencial com alto valor comercial devido à aplicação nas indústrias de higiene, cosméticos e perfumarias. Considerando as características voláteis e estruturas de armazenamento em diferentes órgãos das plantas aromáticas, a determinação de métodos de secagem é de extrema importância e pode afetar tanto o rendimento como a composição do óleo essencial. Este trabalho teve como objetivo principal avaliar o teor e a composição do óleo essencial de patchouli após secagem por diferentes períodos em estufa e a temperatura ambiente. O experimento foi realizado durante o mês de fevereiro de $2011 \mathrm{em}$ delineamento experimental inteiramente casualizado em esquema fatorial $2 \times 6$, correspondente a dois métodos de secagem (estufa $65^{\circ} \mathrm{C}$ e temperatura ambiente) e seis tempos para o início da extração do óleo essencial após a $(0,2,4,6,8$ e 10 dias após a colheita),com três repetições. O óleo essencial foi extraído por meio hidrodestilação em aparelho graduado de Clevenger e os constituintes do óleo essencial foram analisados por cromatografia em fase gasosa acoplada à espectrometria de massas. Houve interação significativa entre os métodos e tempos de secagem tanto para o teor como para a composição do óleo essencial. A secagem das plantas por dois dias em estufa a $65^{\circ} \mathrm{C}$ aumentou o teor de óleo essencial, após este período houve redução significativa. Para as folhas secas em temperatura ambiente, o teor de óleo essencial foi superior aos quatro e aos seis dias após a colheita. Comparando-se os métodos, a secagem em estufa mostrou-se mais eficiente, sendo o teor significativamente superior após dois dias de secagem. Após este período, no entanto, houve drástica redução do teor de óleo essencial, sendo inferior ao teor observado em todos os períodos nas folhas secas em temperatura ambiente. Os diferentes métodos e tempos de secagem alteraram também a composição do óleo essencial. Embora a secagem em estufa a $65^{\circ}$ por dois dias não tenha resultado em redução dos teores dos constituintes majoritários patchoulol e pogostol, maiores teores foram observados após seis ou oito dias de secagem. Quando a secagem foi realizada a temperatura ambiente, não houve alteração dos teores de ambos os constituintes.

Palavras-chave: Pogostemoncablin (Blanco) Benth.,Lamiaceae, secagem, metabolismo secundário, patchoulol, plantas aromáticas.

ABSTRACT. Yield and composition of essential oil of patchouli (Pogostemon cablin (Blanco) Benth.) after different drying periods in oven and at room temperature. Patchouli is an aromatic species belonging to the Lamiaceae family that has essential oil with high commercial value to the hygiene, cosmetic and perfume industries. Considering its volatile characteristics and storage structures in different organs of aromatic plants, the determination of drying methods is extremely important and can affect both the yield and the composition of the essential oil. This work aimed to evaluate the content and composition of the patchouli essential oil after drying it in different periodin an oven and at room temperature. The trial was carried out in February 2011 at an experimental design completely randomized in 2X6 factorial scheme, corresponding to two drying methods (oven at $65^{\circ} \mathrm{C}$ and room temperature) and six periods of essential oil extraction after harvest $(0,2,4,6,8$, and 10 days after harvest), with three replications. The essential oil was extracted by hydrodistillation in a Clevenger apparatus and the essential oil components were analyzed by gas chromatography coupled to mass spectrometry. 
There was significant interaction among the methods and drying times for both the content as well as for the composition of essential oil. The drying of the plants for two days in an oven at $65^{\circ} \mathrm{C}$ increased the essential oil content; after this period, there was a significant reduction. For the leaves that dried at room temperature, their contentof essential oil was superior at the fourth and sixth days after the harvest. When comparing the methods, the drying in the oven was more efficient, with the oil content being significantly higher after two days of drying. After this period, however, there was a drastic reduction in the essential oil content, lower than the one observed in all periods when the leaves dried at room temperature. The different methods and drying periods also altered the essential oil's composition. Although drying in oven at $65^{\circ}$ for two days did not result in reduction of the contents of patchoulol and pogostol, higher contents were observed after six or eight days of drying. When drying was carried out at room temperature, no change in relative content of both components was observed.

Keywords: Pogostemoncablin (Blanco) Benth.,Lamiaceae, drying, secondary metabolism , patchoulol, aromatic plants.

\section{INTRODUÇÃO}

O patchouli (Pogostemoncablin (Blanco) Benth.) é uma espécie aromática pertencente a família Lamiaceae (Souza \& Lorenzi, 2005) cujo óleo essencial possui atividades antifúngica, antihelmintica e anti-tripanossoma (Zhao et al., 2005). Entretanto, o maior valor comercial do óleo essencial desta espécie está relacionado às propriedades de fixação a pele e fragrância amadeirada, conferidas pela presença do constituinte majoritário patchoulol (Sugimura et al., 2005). Deste modo, há grande demanda mundial por parte de indústrias de perfumarias, higiene oral e de cosméticos no uso do óleo essencial de patchouli em suas composições (Zhao et al., 2005; Hsu et al., 2006).

Segundo a International Organization for Standardization (ISO), que normatiza a qualidade dos óleos essenciais, o óleo de patchouli deve apresentar em sua composição os seguintes teores dos constituintes: beta-patchouleno (1,8\%-3,5\%), copaeno (traços- $1 \%$ ), alfa-guaieno $(11 \%-16 \%)$, beta-cariofileno (2\%-5\%), bulneseno (13\%-21\%), nor-patchoulenol $(0,35 \%-1 \%)$ patchoulol $(27 \%$ $35 \%)$, gama-patchouleno (1,8\%-3,5\%) e pogostol (1\%-2,5\%) (ISO, 2002). Deste modo, a realização de pesquisas que avaliem não só o teor do óleo essencial obtido, mas também a sua composição são importantes para assegurar a qualidade do óleo essencial.

De acordo com Reis et al. (2003), logo após a colheita de plantas medicinais inicia-se um processo de ativação enzimática que leva à degradação de suas estruturas e de seus princípios ativos. Desta forma, recomenda-se que logo após a colheita estas plantas sejam secas ou consumidas, diminuindo assim a perda de seus princípios ativos. Para plantas de patchouli, tratamentos de secagem pós-colheita são recomendados, pois promovem aumento significativo na produção de óleo essencial (Guenther, 1972). Ainda, de acordo com o mesmo autor, tal efeito é relacionado à maior permeabilidade das membranas das estruturas que armazenam o óleo essencial desta espécie, permitindo assim, a maior liberação deste durante o processo de extração.

Durante o processo de secagem, outro fator que deve ser avaliado é a velocidade com que a água é retirada do tecido vegetal. Segundo Silva \& Casali (2000), a perda excessivamente rápida dos teores de água pode levar à degradação dos princípios ativos da planta e o inverso pode resultar no crescimento de microrganismos indesejados.

Vários autores têm demonstrado que durante o processo de secagem, o ambiente e as temperaturas as quais as plantas são submetidas podem influenciar o rendimento e a composição do óleo essencial. Rosado et al. (2011) observou em plantas de manjericão (Ocimum basilicum L.) que o processo de secagem em estufa, manteve as características organolépticas de coloração e odor das plantas e aumentou a concentração do constituinte majoritário linalol no óleo essencial. Em plantas de citronela (Cymbopogon winterianus Jowitt) o processo de secagem a $60^{\circ} \mathrm{C}$ durante 48 horas promoveu o maior rendimento de óleo essencial (Rocha et al., 2000). Em plantas de assa peixe (Vermonia polyanthes) a secagem em estufa reduziu o rendimento do óleo essencial quando comparado à secagem à sombra e ao sol (Corrêa et al., 2004).Por outro lado, temperaturas do ar de secagem entre 50 e $60^{\circ} \mathrm{C}$ têm se mostrado eficientes para secagem de um grande número de espécies medicinais independente do método empregado (Melo et al. ,2004).

Tendo em vista o valor econômico do óleo essencial e o fato de trabalhos anteriores terem demonstrado que é possível aumentar o rendimento do óleo essencial de patchouli conforme o manejo realizado na cultura (Costa, 2008; Storck, 2008), 
este trabalho teve como objetivo determinar o método e período de secagem para essa espécie que resulte em maior teor de óleo essencial e níveis de seus constituintes, principalmente o patchoulol.

\section{MATERIAL E MÉTODOS}

O experimento foi realizado no Laboratório de Ecofiosiologia da Universidade Federal do Paraná, Curitiba, PR, no mês de fevereiro de 2011. O delineamento experimental utilizado foi inteiramente casualizado em esquema fatorial 2X6. O esquema fatorial considerou doistipos de secagem das plantas (temperatura ambiente e estufa com circulação de ar forçada) e seis tempos de secagem para o início da extração do óleo essencial: sem secagem (extração com folha fresca logo após a colheita), 2,4,6,8e,10 dias de secagem, com três repetições.

O material vegetal utilizado consistiu de folhas de Pogostemon cablin (Blanco) Benth. (patchouli) coletadas de plantas com aproximadamente um metro de altura e cultivadas na Estação Experimental do Canguiri da Universidade Federal do Paraná, no município de Pinhais, estado do Paraná. Esta região é denominada Primeiro Planalto Paranaense e localiza-se entre as coordenadas $25^{\circ} 25^{\prime}$ latitude sul e $49^{\circ} 08^{\prime}$ longitude oeste, altitude $930 \mathrm{~m}$.

Após a colheita, as plantas de patchouli foram imediatamente levadas ao laboratório de Ecofisiologia onde foram desfolhadas e submetidas aos processos de secagem. Para a secagem em temperatura ambiente o material vegetal foi mantido em bandejas em condições de temperaturas médias entre $23^{\circ} \mathrm{C}$ e $30^{\circ} \mathrm{C}$, e umidade relativa do ar média de $61,6 \%$.

Para a secagem em estufa utilizou-se o modelo FANEM 320-SE com circulação de ar forcada com temperatura ajustada em $65^{\circ} \mathrm{C}$.

A extração do óleo essencial foi realizada por hidrodestilação em aparelho graduado tipo Clevenger. Utilizou-se $50 \mathrm{~g}$ de folhas independente do porcentual de umidade das mesmas no momento da extração. O material vegetal foi então imerso em $1 \mathrm{~L}$ de água destilada em balão de vidro com capacidade para $2 \mathrm{~L}$ durante 5 horas. Para monitorar o teor de umidade das folhas, retirou-se amostras de $20 \mathrm{~g}$ que foram secas em estufa a $65^{\circ} \mathrm{C}$ até massa constante. Após a extração, as amostras de óleo essencial foram quantificadas com base na densidade do óleo e seu teor corrigido para base seca.

A análise das amostras para identificação da porcentagem dos constituintes do óleo essencial foi realizada por meio de cromatografia em fase gasosa (CG) utilizando-se um cromatógrafo Varian modelo CP 3800, com detector de ionização por chama (DIC); coluna capilar HP5 (5\%-fenil95\%-metilsilicone, $30 \mathrm{~m} \times 0,32 \mathrm{~mm} \times 0,25 \mu \mathrm{m})$. As condições de análise foram as seguintes: a) temperatura do injetor: $250^{\circ} \mathrm{C}$, split $1: 20$; b) quantidade de amostra injetada: $1,0 \mu \mathrm{L} ; \mathrm{c}$ ) gás de arraste: hélio a 1,0 mL min-1 na temperatura de $40^{\circ} \mathrm{C}$; d) temperatura do detector DIC: $250^{\circ} \mathrm{C}$; e) programação da temperatura do forno: inicial $60^{\circ} \mathrm{C}$, elevação da temperatura a $240^{\circ} \mathrm{C}$ na razão de $3^{\circ} \mathrm{C}$ por minuto.

Os constituintes do óleo essencial foram identificados por cromatografia em fase gasosa acoplada à espectrometria de massas (CG-EM) em sistema Agilent 5973N; coluna capilar HP5MS (5\%-fenil-95\%-metilsilicone, $30 \mathrm{~m} \times 0,25 \mathrm{~mm} \times 0,25$ $\mu \mathrm{m})$. O detector de massas foi operado no modo de ionização eletrônica a $70 \mathrm{eV}$. A fonte de íons foi operada a $230^{\circ} \mathrm{C}$, o analisador de massas a $150^{\circ} \mathrm{C} \mathrm{e}$ a linha de transferência a $260^{\circ} \mathrm{C}$. A faixa de massas analisada foi de 40 a $450 u$, com 3,15 varreduras por segundo. As demais condições de análise foram às mesmas descritas para a CG.

Os índices de retenção linear foram calculados segundo Van Del Dool \& Kratz (1963) pela injeção de uma série de n-alcanos (C7-C26) na mesma coluna e sob as condições indicadas acima para a análise de CG. A identificação dos constituintes do óleo foi realizada através de pesquisa na espectroteca Wiley 6a edição e pela comparação dos índices de retenção calculados com dados da literatura (Adams, 2007).

As análises de variâncias foram realizadas utilizando-se o programa estatístico ASSISTAT versão 7.4 beta (Silva \& Azevedo, 2006). As variâncias dos tratamentos foram testadas quanto à homogeneidade pelo teste de Bartlett e as médias dos tratamentos comparadas pelo teste de Tukey ao nível de $5 \%$ de probabilidade.

\section{RESULTADOS E DISCUSSÃO}

Os percentuais de umidade das folhas de patchouli no momento da extração do óleo essencial nos diferentes tempos e métodos de secagem são apresentados abaixo (Figura 1).

Houve efeito significativo da interação entre os métodos e tempos de secagem no teor de óleo essencial de folhas de patchouli (Tabela 1). Os resultados obtidos demonstram que a secagem em estufa a $65^{\circ} \mathrm{C}$ e com circulação de ar forçada por dois dias promoveu o maior teor de óleo essencial $\left(88,85 \mu \mathrm{L} . \mathrm{g}\right.$ de massa seca-1) ${ }^{-1}$. Após este período a manutenção das folhas em estufa durante 4, 6, 8 e 10 dias resultou em diminuição para aproximadamente $12,9,5$ e $2 \%$ deste teor, respectivamente. Para as folhas submetidas à secagem em temperatura 


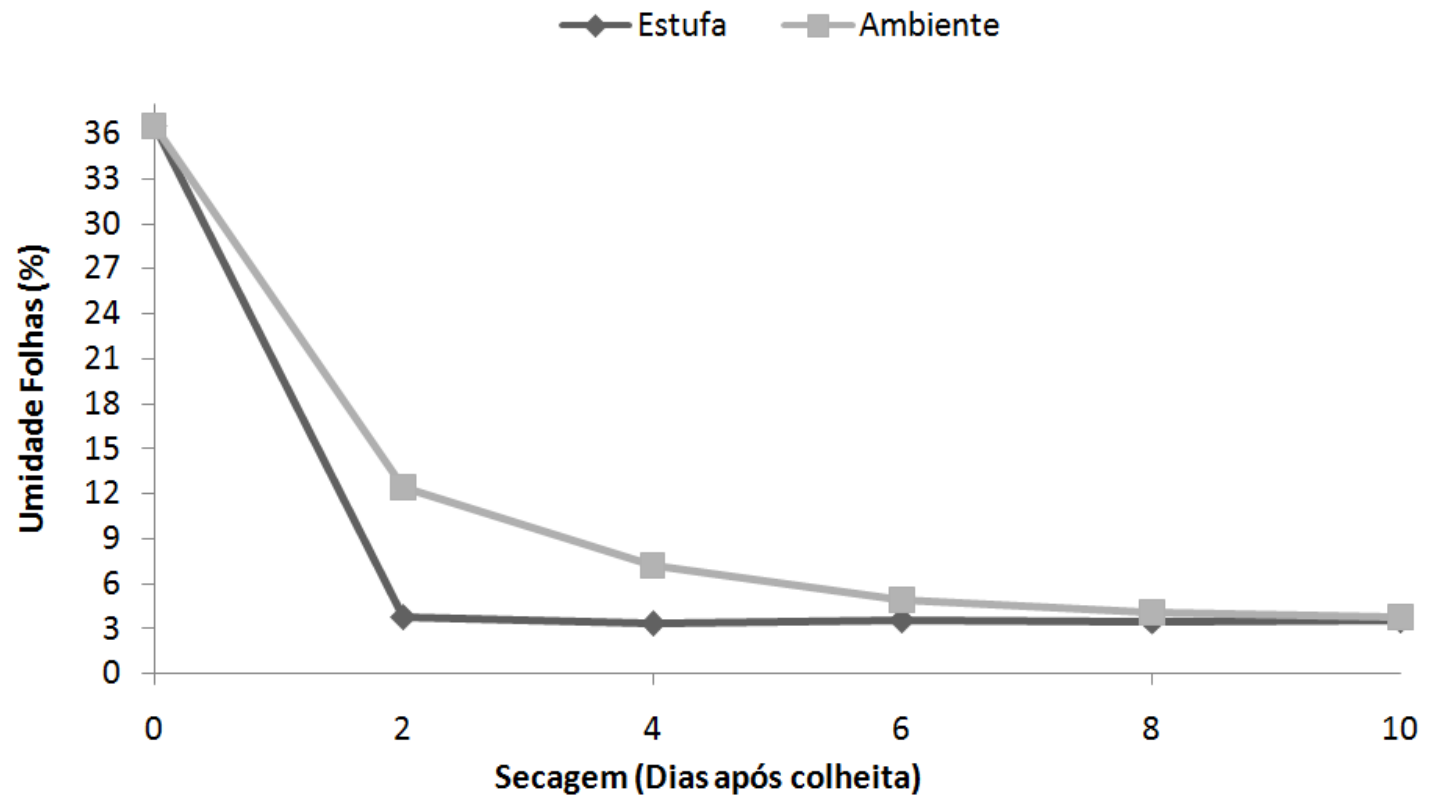

FIGURA 1. Percentual de umidade nas folhas de patchouli no momento da extração do óleo essencial nos diferentes métodos e tempos de secagem. Curitiba, 2011.

ambiente também houve aumento dos teores de óleo essencial, sendo observado, no entanto apósquatroe oitodias. Comparando-se o teor de óleo essencial em ambos os métodos, observou-se que o melhor método para secagem de folhas de patchouli é em estufa por dois dias já que, após este período, este tipo de secagem resulta em redução dos teores.

Para plantas de citronela (Cymbopogon winterianus Jowitt), Rocha et al., (2000) observaram que a secagem das plantas em $60^{\circ} \mathrm{C}$ promoveu teor de óleo essencial estatisticamente igual às plantas secas a 30,40 e $50^{\circ} \mathrm{C}$. Tal fato, do ponto de vista prático é positivo,pois o tempo necessário para secagem na temperatura de $60^{\circ} \mathrm{C}$ foi quatro vezes menor em relação à temperatura de $30^{\circ} \mathrm{C}$. Ainda, segundo os mesmo autores, o maior teor de óleo essencial encontrado nesta temperatura poderia estar relacionado a alterações na permeabilidade ou resistência física-mecânica dos tecidos vegetais, levando a uma rápida evaporação da água livre nos tecidos e menor perda do óleo essencial por volatilização. As estruturas de armazenamento de óleo essencial desta espécie, portanto estariam associadas à células do parênquima clorofiliano e bem protegidas da superfície foliar.

O mesmo fato pode ter ocorrido em plantas de patchouli, uma vez que suas estruturas de armazenamento de óleos essenciais estão, em sua grande maioria, associadas a células do parênquima clorofiliano (Henderson, 1970). A secagem das plantas em $65^{\circ} \mathrm{C}$ pelo período de dois dias pode ter promovido, portanto a desestruturação dos tecidos foliares permitindo deste modo, a maior liberação do óleo essencial do parênquima clorofiliano. A manutenção das plantas por períodos maiores que dois dias a $65^{\circ} \mathrm{C}$, de forma contraria, pode ter promovido a desestruturação das células que armazenam óleo essencial porem levando a volatilização deste durante períodos prolongados de secagem. A secagem em temperatura ambiente por sua vez, pode não ter promovido suficiente desestruturação dos tecidos foliares para liberar

TABELA 1. Rendimento ( $\mu \mathrm{L} . \mathrm{g}$ de massa seca-1) do óleo essencial de patchouli após diferentes períodos (dias após a colheita) e métodos de secagem. Em estufa com circulação de ar forçada a $65^{\circ} \mathrm{C}$ (EST) e em temperatura ambiente (AMB).

\begin{tabular}{ccccccc}
\hline \multirow{2}{*}{ MÉTODO } & \multicolumn{7}{c}{ PERÍODOS } \\
\cline { 2 - 6 } & 0 & 2 & 4 & 6 & 8 & 10 \\
\hline EST & $27,35 \mathrm{Ab}$ & $88,85 \mathrm{Aa}$ & $11,40 \mathrm{Bc}$ & $8,37 \mathrm{Bcd}$ & $4,77 \mathrm{Bde}$ & $2,00 \mathrm{Be}$ \\
AMB & $27,35 \mathrm{Ac}$ & $34,56 \mathrm{Bab}$ & $38,98 \mathrm{Aa}$ & $30,53 \mathrm{Abc}$ & $37,64 \mathrm{Aa}$ & $26,17 \mathrm{Ac}$ \\
\hline
\end{tabular}

Médias seguidas das mesmas letras maiúsculas nas colunas e minúsculas nas linhas não diferem estatisticamente entre só pelo Teste de Tukey ao nível de $5 \%$ de probabilidade.

Rev. Bras. PI. Med., Campinas, v.17, n.4, p.570-576, 2015. 
o óleo essencial armazenado durante o processo de hidrodestilação. Estudos histológicos futuros poderão comprovar esta hipótese.

A análise cromatográfica identificou a presença de 13 diferentes constituintes no óleo essencial (Tabela 2). Com base na literatura pesquisada, os constituintes mais abundantes do óleo essencial de patchouli são: patchoulol, alfa-bulneseno, alfa-guaieno, gama-patchouleno, gama-gurjuneno, germacreno-D, acifileno, 7-epialfa-selineno (Silva et al., 2004; Burè \& Sellier 2004), podendo ocorrer também a presença dos compostos: beta-elemeno, beta-patchouleno, cariofileno, alfa-patchouleno e seicheleno (Hu et al., 2006; Singh et al., 2002; Zhu et al., 2003).

Com exceção dos compostos gama- patchouleno, gama-gurjuneno, germacreno-D e 7-epi-alfa-selineno, os demais foram encontrados na análise do óleo essencial do material vegetal utilizado neste trabalho. Além destes, foram encontrados também os compostos pogostol, nor-patchoulenol, óxido de cariofileno, linalol e 1-8 cineol.

Em relação às concentrações dos compostos do óleo essencial, foi possível observar que a secagem em estufa por dois dias proporcionou aumento das concentrações de alfa-bulneseno, acifileno, nor-patchoulenol, (E)-cariofileno e cicloseicheleno. Aos quatro e aos seisdias houve aumento do constituinte nor-patchoulenol e, patchoulol + pogostol, respectivamente. A secagem das plantas por 10 dias em estufa reduziu

TABELA 2. Composição química do óleo essencial de patchouli após diferentes tempos (dias após a colheita) e métodos de secagem. Em estufa com circulação de ar forçada a $65^{\circ} \mathrm{C}$ (EST) e em temperatura ambiente (AMB).

\begin{tabular}{|c|c|c|c|c|c|c|c|}
\hline \multirow{2}{*}{ COMPOSTOS } & \multirow{2}{*}{ MÉTODO } & \multicolumn{6}{|c|}{ PERÍODOS } \\
\hline & & 0 & 2 & 4 & 6 & 8 & 10 \\
\hline Patchoulol + & EST & $68,63 \mathrm{Abc}$ & $66,74 \mathrm{Ac}$ & $69,83 \mathrm{Abc}$ & $79,12 \mathrm{Aa}$ & 75,06 Aab & $57,33 \mathrm{Bd}$ \\
\hline Pogostol & AMB & $68,63 \mathrm{Aa}$ & 63,71 Aa & $66,31 \mathrm{Aa}$ & $66,61 \mathrm{Ba}$ & $66,62 \mathrm{Ba}$ & $65,62 \mathrm{Aa}$ \\
\hline \multirow{2}{*}{ a-Bulneseno } & EST & $6,91 \mathrm{Abc}$ & $8,66 \mathrm{Aa}$ & 7,97 Aab & $5,39 \mathrm{Bcd}$ & $3,81 \mathrm{Bd}$ & $1,20 \mathrm{Be}$ \\
\hline & AMB & $6,91 \mathrm{Ab}$ & 8,43 Aab & $8,15 \mathrm{Aab}$ & $8,96 \mathrm{Aa}$ & 8,69 Aa & $9,20 \mathrm{Aa}$ \\
\hline a-Humuleno + & EST & $4,10 \mathrm{Aa}$ & $2,99 \mathrm{Bb}$ & $2,56 \mathrm{Bc}$ & $1,89 \mathrm{Bd}$ & 2,01 Bcd & $0,47 \mathrm{Be}$ \\
\hline a-Patchouleno & AMB & $4,10 \mathrm{Aa}$ & 3,39Ab & $3,12 \mathrm{Ab}$ & $3,49 \mathrm{Ab}$ & $4,45 \mathrm{Aa}$ & $3,26 \mathrm{Ab}$ \\
\hline \multirow{2}{*}{ a-Guaieno } & EST & $10,75 \mathrm{Aa}$ & 10,01 Aab & $8,82 \mathrm{Bb}$ & $5,83 \mathrm{Bc}$ & $3,98 \mathrm{Bc}$ & 2,01 Bd \\
\hline & AMB & $10,75 \mathrm{Aa}$ & $10,29 \mathrm{Aa}$ & $10,22 \mathrm{Aa}$ & $10,31 \mathrm{Aa}$ & $10,60 \mathrm{Aa}$ & $11,02 \mathrm{Aa}$ \\
\hline \multirow{2}{*}{ Acifileno } & EST & $0,47 \mathrm{Ad}$ & $1,52 \mathrm{Aa}$ & 1,38 Aab & $1,17 \mathrm{Bb}$ & $0,81 \mathrm{Bc}$ & 0,20 Be \\
\hline & AMB & $0,47 \mathrm{Ab}$ & $1,36 \mathrm{Aa}$ & $1,37 \mathrm{Aa}$ & $1,42 \mathrm{Aa}$ & $1,55 \mathrm{Aa}$ & $1,60 \mathrm{Aa}$ \\
\hline \multirow{2}{*}{ Nor-Patchoulenol } & EST & $0,81 \mathrm{Ab}$ & $1,41 \mathrm{Aa}$ & $1,51 \mathrm{Aa}$ & 1,44 Ba & $1,32 \mathrm{Ba}$ & $0,53 \mathrm{Bc}$ \\
\hline & AMB & $0,81 \mathrm{Ad}$ & $1,17 \mathrm{Bc}$ & $1,39 \mathrm{Ab}$ & $1,69 \mathrm{Aa}$ & 1,50 Aab & $1,68 \mathrm{Aa}$ \\
\hline \multirow{2}{*}{ (E)-Cariofileno } & EST & $1,26 \mathrm{Ac}$ & $1,98 \mathrm{Aa}$ & 1,53 Bb & $0,96 \mathrm{Bd}$ & $0,16 \mathrm{Be}$ & $0,12 \mathrm{Be}$ \\
\hline & AMB & $1,26 \mathrm{Ac}$ & $2,06 \mathrm{Ab}$ & 2,13 Aab & $2,16 \mathrm{Aab}$ & $2,18 \mathrm{Aab}$ & 2,24 Aa \\
\hline \multirow{2}{*}{ Cicloseicheleno } & EST & $0,13 \mathrm{Ab}$ & $0,20 \mathrm{Ba}$ & $0,08 \mathrm{Bc}$ & 0,01 Bd & $0,19 \mathrm{Bc}$ & 0,05 Bd \\
\hline & AMB & $0,13 \mathrm{Ab}$ & $0,25 \mathrm{Aa}$ & $0,21 \mathrm{Aa}$ & $0,12 \mathrm{Ab}$ & $0,01 \mathrm{Ac}$ & $0,04 \mathrm{Ac}$ \\
\hline \multirow{2}{*}{$\beta$-Elemeno } & EST & $0,38 \mathrm{Aa}$ & $0,38 \mathrm{Ba}$ & $0,27 \mathrm{Bb}$ & 0,01 Bd & $0,19 \mathrm{Bc}$ & 0,05 Bd \\
\hline & AMB & $0,38 \mathrm{Ac}$ & $0,60 \mathrm{Aa}$ & $0,46 \mathrm{Ab}$ & $0,47 \mathrm{Ab}$ & $0,47 \mathrm{Ab}$ & $0,48 \mathrm{Ab}$ \\
\hline \multirow{2}{*}{$\beta$-Patchouleno } & EST & $0,46 \mathrm{Ae}$ & $0,89 \mathrm{Ab}$ & $0,70 \mathrm{Bc}$ & $0,55 \mathrm{Bd}$ & $2,05 \mathrm{Aa}$ & $0,60 \mathrm{Bd}$ \\
\hline & AMB & $0,46 \mathrm{Ae}$ & $0,79 \mathrm{Bcd}$ & $0,86 \mathrm{Abc}$ & $0,78 \mathrm{Ad}$ & $0,87 \mathrm{Bb}$ & 0,95 Aa \\
\hline \multirow{2}{*}{ Oxido de Cariofileno } & EST & $1,82 \mathrm{Aa}$ & $0,12 \mathrm{Bd}$ & $0,36 \mathrm{Ac}$ & $0,34 \mathrm{Ac}$ & $0,49 \mathrm{Ab}$ & $0,18 \mathrm{Ad}$ \\
\hline & AMB & $1,82 \mathrm{Aa}$ & $0,66 \mathrm{Ab}$ & $0,01 \mathrm{Bc}$ & $0,01 \mathrm{Bc}$ & $0,01 \mathrm{Bc}$ & $0,01 \mathrm{Bc}$ \\
\hline
\end{tabular}

Médias seguidas das mesmas letras maiúsculas nas colunas e minúsculas nas linhas não diferem estatisticamente entre só pelo Teste de Tukey ao nível de $5 \%$ de probabilidade.

Rev. Bras. PI. Med., Campinas, v.17, n.4, p.570-576, 2015. 
significativamente os teores de todos os constituintes do óleo essencial, com exceção do constituinte 1-8 cineol que teve seu teor aumentado.

Para as folhas submetidas à secagem em temperatura ambiente, as concentrações dos constituintes majoritários patchoulol + pogostol não foram alteradas. Após dois dias, no entanto, houve aumento da concentração dos constituintes acifileno, cicloseicheleno e beta-elemeno. Aos quatro dias, os constiuintes que apresentaram aumento em suas concentrações foram acifileno e cicloseicheleno. Os constituintes alfa-bulneseno, acifileno, linalol e 1-8 cineol; e alfa-bulneseno, alfa-patchouleno+ alfahumuleno e acifileno, por sua vez, tiveram os maiores teores aos seise aos oitodias respectivamente. Aos 10 dias de secagem em temperatura ambiente os constituintes alfa-bulneseno, acifileno, norpatchoulenol e (E)-cariofileno foram os que tiveram maiores concentrações no óleo essencial.

A principal causa da diversidade de terpenos está relacionada à presença de diversas enzimas regulatórias envolvidas na síntese dos mesmos e que atuam na presença de vários substratos. A redução da síntese de um ou mais produtos é correlacionada, portanto,com a disponibilidade de substrato para a síntese de outros compostos já que uma única enzima pode levar à formação de vários terpenos utilizando um único substrato (Degenhardt et al. 2009).

Em trabalhos anteriores realizados no Brasil com patchouli foram obtidas concentrações de patchoulol em níveis superiores aos descritos pela ISO (Sant'Ana et al., 2010; Silva et al., 2004; Storck, 2008). Tal fato também foi encontrado neste experimento em todos os tratamentos e, portanto recomenda-se que a extração do óleo essencial de patchouli seja afeita após dois dias de secagem em estufa a $65^{\circ} \mathrm{C}$. Nestas condições, além do tempo de secagem das plantas ser inferior aos demais tratamentos, obtem-se teor de óleo essencial superior e com concentração de patchoulol elevada.

\section{CONCLUSÕES}

A secagem de plantas de patchouli durante dois dias em estufa a $65^{\circ} \mathrm{C}$ promove maior teor do óleo essencial, e mantém níveis satisfatórios do constituinte patchoulol em sua composição. A secagem em estufa a $65^{\circ} \mathrm{C}$ por períodos superiores a dois dias promove redução drástica do teor de óleo essencial em folhas de patchouli. Caso a secagem em temperatura ambiente seja a única alternativa para o produtor, a extração do óleo essencial deverá ser realizada após quatro dias da colheita.

\section{AGRADECIMENTOS}

Os autores agradecem a CAPES pela bolsa concedida ao primeiro autor e ao CNPq pela bolsa de produtividade do segundo autor.

\section{REFERÊNCIAS}

ADAMS, R.P. Identification of Essential Oil Components by Gas Chromatography / Mass Spectrometry, 4th ed. Carol Stream, IL: Allured Publishing Corp., 2007, 804p.

BURE,C..M.; SELLIER, N.M. Analysis of the essential oil of Indonesian patchouli (Pogostemon cablin Benth.) using GC/MS (El/Cl). Journal of Essential Oil Research, Carol Stream, v. 16, n.1, p. 17-19, 2004.

CORREAA, R. M. et al. Rendimento de óleo essencial e caracterização organoléptica de folhas de assa-peixe submetidas a diferentes métodos de secagem. Ciência e Agrotecnologia,v. 28, n. 2, p. 339-344, 2004.

COSTA, A. G. Desenvolvimento vegetativo, rendimento e composição do óleo essencial de patchouli após adubação nitrogenada. 2008. 92f. Dissertação (Mestrado em Agronomia). Universidade Federal do Paraná, Curitiba.

DEGENHARDT, J. et al. Monoterpene and sesquiterpene synthases and the origin of terpene skeletal diversity in plants. Phytochemistry,v. 70. p. 1621-1637, 2009.

GUENTHER, E. Oil of patchouly. In: GUENTHER, E. Essential oils of the plant family Labiatae. New York: Krieger Publishing Company, 1972. p. 552-575.

HENDERSON, W. et al. Chemical and morphological studies on sites of sesquiterpene accumulation in Pogostemon cablin (Patchouli). Phytochemistry, v. 9, p. 1219-1228, 1970.

HU, L.F. et al. GC-MS. Fingerprint of Pogostemoncablin in China. Journal of Pharmaceutical and Biomedical Analysis,v.42.p. 200-206, 2006.

HSU, H.C. et al. a -Bulnesene, a novel PAF receptor antagonist isolated from Pogostemon cablin. Biochemical and Biophysical Research Communications,v. 345, n.7, p.1033-1038, 2006.

MELO, E.C. et al. Influencia do processo de secagem na qualidade de plantas medicinais - Revisão. Engenharia na Agricultura, v.12, n.4, p. 307-315, 2004.

REIS, M.S.; MARIOT, A.; STEENBOCK, W. Diversidade e domesticação de plantas medicinais. In: SIMÕES, C.M.O et al. Farmacognosia: da planta ao medicamento. 5. ed. Porto Alegre/Florianópolis: Editora UFRGS/ Editora UFSC, 2003. p.43-74.

ROCHA, S.F.R. et al. Influência de cinco temperaturas de secagem no rendimento e composição do óleo essencial de citronela (Cymbopogon winterianus Jowitt). Revista Brasileira de Plantas Medicinais, v.3, n.1, p. 73-78, 2000.

ROSADO, L. D. S. et al. Influência do processamento da folha e tipo de secagem no teor e composição química do óleo essencial de manjericão cv. Maria Bonita. Ciência e Agrotecnologia, v. 35, n. 2, p. 291-296, 2011.

SANT'ANA, T.C..P de. et al. Influência do armazenamento de folhas secas no óleo essencial de patchouli (Pogostemon cablin Benth.). Química Nova, v. 33, n. 
6, p. 1263-1265, 2010.

SILVA, F. de A.S.; AZEVEDO, C.A.V. de. A new version of the assistat-statistical assistance software. In: World Congress on Computers in Agriculture, 4. Orlando-FLUSA: Anais: Orlando: American Society of Agricultural Engineers, p.393-396, 2006.

SILVA, F. da; CASALI, V.W.D. Plantas medicinais e aromáticas: Pós-colheita e óleos essenciais. Viçosa, UFV/DFT, $2000.135 \mathrm{p}$.

SILVA, M.A.S. et al. Composition and chemical variation during daytime of constituents of the essential oil of Pogostemon patchouli pellet leaves. Acta Horticulturae, n. 629, p. 145-147, 2004.

SINGH, M. et al. Herbage, oil yield and oil quality of patchouli [Pogostemoncablin (Blanco) Beth.] influeced by irrigation, organic mulch and nitrogen aplication in semi-arid tropical climate. Industrial Crops and Products, v. 16, p. 101-107, 2002.

SOUZA, V.C; LORENZI, H. Sistemática Botânica. Guia ilustrado para a identificação das famílias de
Angiospermas da flora brasileira, baseado em APGII. Nova Odessa, SP: Instituto Plantarum, 639 p., 2005.

STORCK, R. C. Sombreamento, ácido giberélico e extrato de alaga marinha no desenvolvimento e produção de óleos essenciais em patchouli. 2008. 98 f. Dissertação (Mestrado em agronomia). Universidade Federal do Paraná, Curitiba.

SUGIMURA, Y. et al.Transgenic patchouli plants produced by Agrobacterium-mediated Transformation. Plant Cell Tissue and Organ Culture, Urbana, v. 82, n.3, p.251-257, 2005.

VAN DEN DOOL, H.; KRATZ, D. P.D.J.A. Generalization of the retention index system including linear temperature programmed gas-liquid partition chromatography. Journal of Chromatography,v.11, p. 463-71, 1963.

ZHAO, Z. et al. Determination of Patchoulic Alcohol in Herba Pogostemonis by GC-MS-MS. Chemical Pharmaceutical Bulletin, v. 53, n.7, p. 856-860, 2005. 